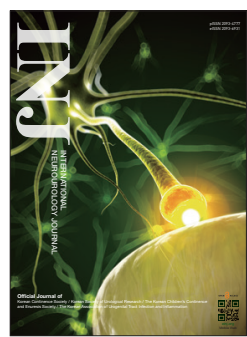

\title{
Strategies for Overcoming Intractable Disorders or Memory Impairments
}

\author{
Chang-Ju Kim (iD https://orcid.org/0000-0003-4749-5795 \\ Department of Physiology, College of Medicine, Kyung Hee University, Seoul, Korea \\ Email: changju@khu.ac.kr
}

The topic of this issue of the International Neurourology Journal is "Strategies for overcoming intractable disorders or memory impairments." This topic is intended to examine the mechanisms underlying the occurrence of intractable diseases or memory impairments, and to provide methods for remedial treatment or symptom relief accordingly. There are 2 review papers and 3 original papers, which describe 2 treatment strategies involving exercise and 1 involving medication. These 5 articles address a wide range of subjects: bladder fibrosis, intractable epilepsy, particular matter $\left(\mathrm{PM}_{10}\right)$-induced trachea inflammation, aging-induced sarcopenia, and amyloid- $\beta$-induced Alzheimer disease.

Kim et al. [1] wrote a review paper describing bladder fibrosis as a condition involving irreversible changes in the bladder that is associated with underactive bladder and impaired bladder compliance. They pointed out that bladder fibrosis is a serious problem, for which it is difficult to determine the exact underlying mechanism and risk factors. Due to the unknown nature of bladder fibrosis-related disorders, the progression of urination problems related to bladder fibrosis cannot be predicted. Therefore, it is difficult to prevent and treat bladder fibrosis.

Moon and Cho [2] discussed epileptic encephalopathy, which is a fatal pediatric disease characterized by medically resistant seizures that can contribute to a global delay in development. In this review, they introduced the molecular mechanisms that cause excitatory/inhibitory imbalances in the most common epileptic encephalopathies in pediatric patients. They discussed voltage-gated ion channels, synaptic transduction-re- lated proteins, and ligand-gated ion channels in relation to the pathophysiology of Ohtahara syndrome, Dravet syndrome, and Lennox-Gastaut syndrome. Finally, they reviewed the antiepileptic drugs used to treat seizures in patients with epileptic encephalopathy.

Inhalation of air containing large amounts of particular matter causes various respiratory diseases, including asthma, chronic obstructive pulmonary disease, and lung cancer. Hwang et al. [3] studied changes in the expression of inflammatory factors in response to polydeoxyribonucleotide administration in a $\mathrm{PM}_{10}$-exposed trachea inflammation model. Polydeoxyribonucleotide treatment attenuated inflammation in the trachea of $\mathrm{PM}_{10}$-exposed mice. They concluded that this favorable effect of polydeoxyribonucleotide was due to activation of the adenosine $\mathrm{A}_{2 \mathrm{~A}}$ receptor via the cyclic adenosine monophosphate-protein kinase A pathway.

The next 2 papers are on exercise. Ko and Ko [4] evaluated the protective effect of voluntary wheel running exercise on muscle loss and muscle weakness in the gastrocnemius of old rats. Aging-induced decreases in muscle mass, strength, and coordination were associated with diminished activity of the peroxisome proliferator-activated receptor-gamma coactivator1a/fibronectin type III domain-containing protein 5/adenosine monophosphate-activated protein kinase (PGC-1 $\alpha /$ FNDC5/ AMPK) signaling pathway in the gastrocnemius. Voluntary wheel running enhanced vascular endothelial growth factor expression by activating the PGC-1 1 /FNDC5/AMPK signaling pathway, leading to increased muscle mass, strength, and coor- 


\section{dination.}

Kim et al. [5] investigated the effect of treadmill running on spatial learning memory in terms of activation of the nuclear factor kappa $\mathrm{B}(\mathrm{NF}-\mathrm{\kappa} \mathrm{B})$ and mitogen-activated protein kinase (MAPK) signaling pathway in Alzheime disease rats. They also evaluated the effect of treadmill running on pro-inflammatory cytokine production and apoptosis, and found that treadmill running inactivated the $\mathrm{NF}-\kappa \mathrm{B} / \mathrm{MAPK}$ signaling pathway and suppressed proinflammatory cytokine production. Treadmill running also inhibited apoptosis and improved spatial learning memory.

This special issue will be an opportunity to discover new treatment techniques for intractable urological diseases and mechanisms of disease development. On behalf of the editorial board, I am grateful to the authors for contributing their valuable articles to this issue.

- Conflict of Interest: No potential conflict of interest relevant to this article was reported.

\section{REFERENCES}

1. Kim SJ, Kim J, Na YG, Kim KH. Irreversible bladder remodeling induced by fibrosis. Int J Neurourol J 2021;25(Suppl 1):S3-7.

2. Moon JU, Cho KO. Current pharmacologic strategies for treatment of intractable epilepsy in children. Int J Neurourol J 2021;25(Suppl 1):S8-18.

3. Hwang L, Jin JJ, Ko IG, Kim S, Cho YA, Sung JS, et al. PDRN attenuates airway inflammation through $\mathrm{A}_{2 \mathrm{~A}} \mathrm{R}$ signaling pathway in $\mathrm{PM}_{10}$-exposed mice. Int J Neurourol J 2021;25(Suppl 1):S19-26.

4. Ko YJ, Ko IG. Voluntary wheel running exercise improves aginginduced sarcopenia via activation of PGC-1a/FNDC5/AMPK signaling pathway. Int J Neurourol J 2021;25(Suppl 1):S27-34.

5. Kim SH, Ko YJ, Kim JY, Sim YJ. Treadmill running improves spatial learning memory through inactivation of NF-KB/MAPK signaling pathway in amyloid- $\beta$-induced Alzheimer's disease rats. Int J Neurourol J 2021;25(Suppl 1):S35-43. 